\title{
Study on accuracy of casting flow simulation
}

\author{
*Youngsim Choi ${ }^{1,2}$, Jun-Ho Hong ${ }^{2}$, Ho-Young Hwang ${ }^{2}$ and Kyuhong Kim ${ }^{1,3}$ \\ 1. Dept. of Mechanical \& Aerospace Engineering, Seoul Nat. Univ., Republic of Korea \\ 2. Korea Institute of Industrial Technology (KITECH), Republic of Korea \\ 3. Institute of Advanced Aerospace Technology, Seoul Nat. Univ., Republic of Korea
}

\begin{abstract}
In the field of casting flow simulation, the application of body-fitted coordinate (BFC) has not been widely used due to the difficulty and low efficiency of grid generation, despite the availability of good quality analysis results. Cartesian coordinates, on the other hand, have been used predominantly in casting process simulations because of their relatively easy and fast grid generation. However, Cartesian grid systems cannot obtain accurate results because they cannot express the geometries properly. In this study, Cut Cell method was applied to solve this problem. The three-dimensional incompressible viscous governing equation was analyzed using a function defined for the volume and area of the casting in the cutting cell. Using the Cut Cell method, accurate flow analysis results were also obtained in the Cartesian grid systems. The tests of simple shape and the applications of actual casting product have been tried with Cut Cell method.
\end{abstract}

Key words: casting flow; Cut Cell; Cartesian coordinates; body-fitted coordinates; filling pattern; conservation of momentum
CLC numbers: TP391.99
Document code: A
Article ID: 1672-6421(2017)05-333-06

$\mathrm{T}$ he first step when analyzing numerical properties in computational fluid dynamics (CFD) is to generate a grid in the computational domain. In order to obtain the exact value of the flow fields, it is crucial to generate an appropriate grid that fits the shape to be analyzed ${ }^{[1]}$. To accomplish this, body-fitted coordinate (BFC) systems were used. These grid systems can show exactly the surface of the body to be calculated, and appropriate analytical values can be obtained using these systems. However, these systems require significant effort to generate grids that fit the body ${ }^{[2]}$. Skilled techniques are required to create the proper grids, and a lot of time is required to generate the grids; there are times when the time required to generate the grids exceeds the computational time for the flow simulation. The casting process runs very quickly, so the calculation time is an element that is just as important as the accuracy of casting flow analysis. In addition, as most casting products are complex geometries with many slopes and curves, it is very difficult to apply the BFC method in casting flow simulation ${ }^{[3,4]}$. For such reasons, the

\section{*Youngsim Choi}

Female, born in 1979, Senior Researcher. Her research interests mainly focus on the numerical scheme of mold filling simulation and the optimal gating system design.

E-mail: raycute@kitech.re.kr

Received: 2017-08-15; Accepted: 2017-08-29
Cartesian coordinate systems were normally used in the casting flow analysis field. These coordinate systems generate rectangular shaped grids (in the case of two dimensions) regardless of the appearance of the body, making it easy to generate grids without any particular skill, and it does not take a long time to generate the grids as well. If there are both castings and molds in a cell, it is determined as a casting or mold cell depending on the volume ratio of the casting in the cell. When generating grids of products with slopes or curves, grids are generated in the stepped shape, and for such reasons, the generated grids on Cartesian coordinates are not consistent with the original geometries (Fig. 1). Stair grids that do not match the body have a negative effect on the flow analysis results. For example, there is a loss of momentum, which results in a loss of velocity, and incorrect filling behavior ${ }^{[5]}$. When using the Cartesian coordinate systems to solve these problems, the number of grids was increased so that the body could be expressed as accurately as possible. The increase in the number of grids can solve minimal problems caused by a distorted shape, but it cannot resolve fundamental problems that occur due to the curves or diagonals of the original body being changed into stair-forms on grid systems. In order to resolve these problems, the partial cell treatment (PCT) based on Cut Cell method was applied for casting flow simulations. The PCT is used to analyze fluids flowing around a moving object in the 

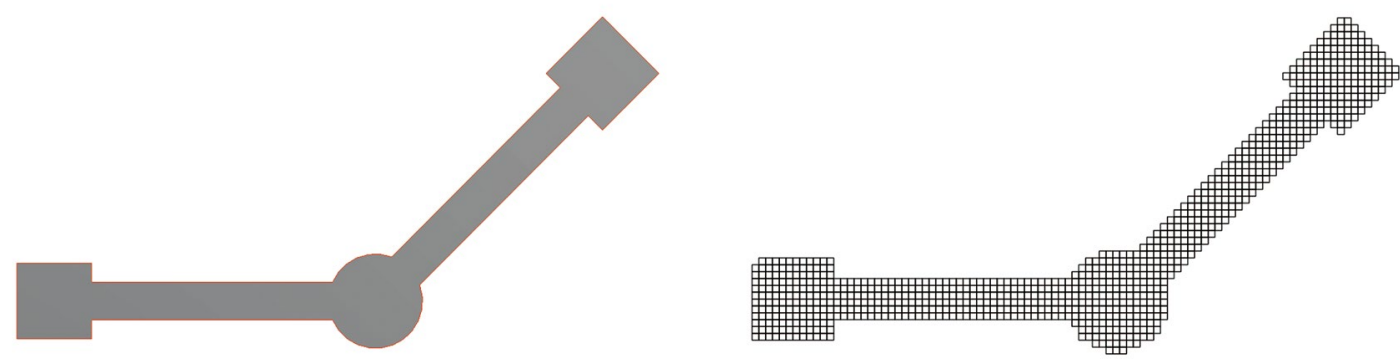

Fig. 1: Original shape (left) and generated meshes (right)

Cartesian coordinate systems ${ }^{[6]}$. The Cut Cell method considers the governing equations with the ratio in a cell that accounts for the body cut by the Cartesian coordinates to obtain similar results as that of the body-fitted coordinates. This method maintains the advantages of the Cartesian coordinate systems (convenience and speed for generating grids), while also being able to obtain accurate flow analysis results. The developed Cut Cell method was tested for simple problems and was applied in actual casting products.

\section{Numerical method}

\subsection{Cut Cell method}

Figure 2 shows that the meshes for the model with a circular shape were created in the Cartesian coordinates. Here, the meshes that include both casting and mold are defined as Cut Cells.

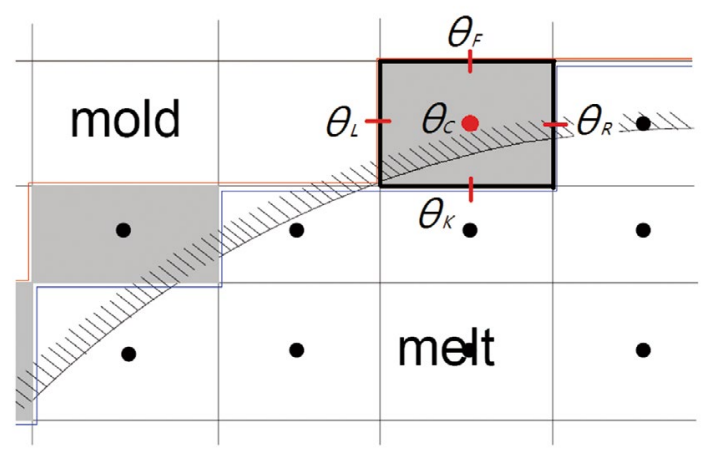

Fig. 2: Cut cells on surface of a circle

In the cut cell, an openness function $\theta$ defined for the volume and the area of castings is used. When $\theta=0$, it shows the mold part cell. When $\theta=1$, it shows the cell of the casting part. When $0<\theta<1$, it shows the cell of the interface between casting and mold. Such a definition is very similar to the method for the Volume of Fluid (VOF) function defining the free surface interface of molten metal. The difference is that function $\theta$ is used not only for the center of the grid, but also defining the ratio of the casting in the grid interfaces as well. In the Cartesian coordinates, the grids are divided into casting and mold. In other words, they are divided into the flow calculation area and non-calculation area, but in the Cut Cell method, $\theta$ is used to calculate the grids treated as non-calculation areas. When taking a look at the cell indicated with bold lines in Fig. 2, $\theta_{c}$ shows the volume fraction of the casting at the center of the cell, and that value is used in the continuity equation and VOF equation. $\theta_{R}$ and $\theta_{L}$ of the $x$ axis and $\theta_{F}$ and $\theta_{K}$ of the $y$ axis show the area fraction of the casting on each of the cell faces. In the case of three dimensions, $\theta_{Z}$ and $\theta_{B}$ of the $z$ axis direction were added. These values show the location of the body in the grids and are used in the momentum equation. Using the values of $\theta$, the conservation of mass is calculated for the amount that the actual casting takes up in a cell and the VOF equation is made to calculate the free surface of the molten metal flow.

In the Cut Cell method, $\theta$ is a variable that shows the original geometrical surface shape information in the calculating grids. Using these values, it is possible to conserve losses of momentum that occur due to the changed shapes or correcting the overload of pressure on the wall boundaries. These values are used in the computation of velocities that are defined on the cell faces. The values of $\theta$ are found through the mesh generator that was developed by our group, and these were used for analysis.

\subsection{Actual shape mapping}

In the Cartesian coordinates, it is evident that the shape of generated meshes can be expressed as a stair-form for curved areas, as shown in Fig. 3 (a). The cut cell value is extracted through preprocessing (the generation of grids) and it is used in the Cut Cell method for fluid flow analysis. In addition, the Marching Cubes algorithm ${ }^{[7]}$ was used to extract the iso-surface using the cell unit filling value and then perform a specific body face extraction and clipping to perform graphical postprocessing (named the Actual Shape Mapping). It is possible to confirm visible effects such as actual shapes rather than stepped grids [Fig. 3 (b)].

\subsection{Governing equations}

The three-dimensional flow field of incompressible viscous fluid is governed by the Navier-Strokes equations. The continuity equation, momentum equation, and Volume of Fluid (VOF) equation for coupling with the function $\theta$ are shown below:

Continuity equation:

Momentum equation:

$$
\nabla \cdot(\theta \vec{u})=0
$$

$$
\frac{\partial(\theta \vec{u})}{\partial t}+\nabla \cdot(\theta \vec{u} \vec{u})=-\frac{\theta}{\rho} \nabla p+\nu \theta \nabla^{2} \vec{u}+\theta \vec{g}
$$

Volume of fluid equation: 

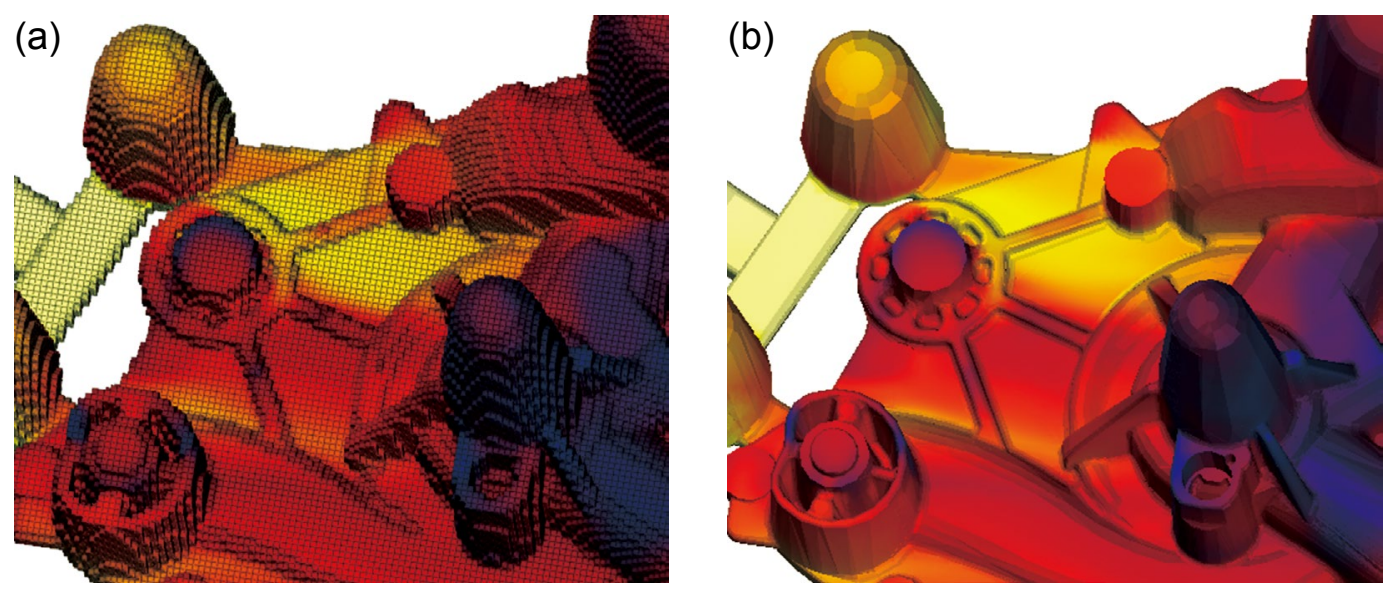

Fig. 3: Stair-form grids (a) and Actual Shape Mapping (b)

$$
\frac{\partial(\theta f)}{\partial t}+\nabla \cdot(\theta f \vec{u})=0
$$

where $\vec{u}$ is the velocity vector and $\vec{g}$ is the acceleration of gravity, $v$ is the kinematic viscosity, $\rho$ is the density of molten metal, $p$ is pressure, $f$ is the volume fraction of fluid, and $t$ is time.

Equations (1) and (3) are differentiated as follows in the staggered grid system:

$$
\begin{gathered}
\frac{u_{i+1 / 2, j, k} \theta_{R}-u_{i-1 / 2, j, k} \theta_{L}}{\Delta x}+\frac{v_{i, j+1 / 2, k} \theta_{F}-v_{i, j-1 / 2, k} \theta_{K}}{\Delta y} \\
+\frac{w_{i, j, k+1 / 2} \theta_{T}-w_{i, j, k-1 / 2} \theta_{B}}{\Delta z}=0 \\
f_{i, j, k}^{n+1} \theta_{C}=f_{i, j, k}^{n} \theta_{C}+\frac{\Delta t}{\Delta x}\left[f u_{i-1 / 2, j, k} \theta_{L}-u_{i+1 / 2, j, k} \theta_{R}\right] \\
+\frac{\Delta t}{\Delta y}\left[f v_{i, j-1 / 2, k} \theta_{K}-f v_{i, j+1 / 2, k} \theta_{F}\right] \\
+\frac{\Delta t}{\Delta z}\left[f w_{i, j, k-1 / 2} \theta_{B}-f w_{i, j, k+1 / 2} \theta_{T}\right]
\end{gathered}
$$

where $u, v$ and $w$ are $x$-, $y$ - and $z$-component of the velocity vector and the subscripts $i, j$ and $k$ indicate the point of $i-, j$ - and $k$-th in the grid system.

In equation (2), for the differentiation of the convection term, a hybrid scheme was used to increase the numerical computational accuracy.

When applying the Cut Cell method in the governing equations, if the $\theta$ has sufficiently small value, it can cause an increase in numerical instability, and therefore, it is advised to set the $\theta=0$ for analysis.

For flow fields, the SOLA (a Solution Algorithm for Transient Fluid Flow) method ${ }^{[8]}$ was used, and the VOF method was used to track the free surface interface of the molten metal.

\section{Numerical results and discussion}

The Cut Cell method is applied to the following cases. First, simple problems were tested for verifying the developed module. After verification, more complicated real casting products were simulated. No temperature was taken into account for focusing on filling patterns.

\subsection{Test case 1}

In order to verify the developed code, a simple body analysis was carried out. This product has two runners lying at an angle of 120 degrees and the molten metal, through the sprue, passes the runners and enters the casting cavity. The grid interval was set at $1 \mathrm{~mm}$. The material of the molten metal was A356 $(\mathrm{AC} 4 \mathrm{CH})$ and the material of the mold was H13 (SKD61), and the molten metal pouring velocity was set at $30 \mathrm{~cm} \cdot \mathrm{s}^{-1}$. Figure 4 shows the molten metal filling rate at $22 \%, 30 \%$ and $50 \%$ from the $z$-cross section. When using the traditional method with no Cut Cell treatment [Fig. 4 (a)], the molten metal flow velocity in the slanted runner on an orthogonal coordinate axis was slower than the velocity of the runner lying vertically on the axis. On the other hand, when using the Cut Cell method [Fig.4 (b)], the molten metal progression velocity of the two runners was the same. The grids of the slanted runner on the axis are not consistent with the original shape. These appear as the grids in the stair-form. If the momentum in the cutting cell at the interface between the casting and mold is not preserved by using function, there will be momentum loss. This causes a loss in velocity and can cause velocity differences between the two runners.

\subsection{Test case 2}

A casting product in the shape of a half sphere was analyzed using the sand casting process. The material of the molten metal was $\mathrm{A} 356(\mathrm{AC} 4 \mathrm{CH})$ and the material of the mold was furan resin bonded sand. The pouring velocity of molten metal at the sprue was set at $100 \mathrm{~cm} \cdot \mathrm{s}^{-1}$. Figure 5 shows the molten metal filling rate at $20 \%, 40 \%$ and $96 \%$, and it is the analysis result by applying the actual shape mapping.

Figure 5 (a) shows the analysis results with the traditional method, and Fig. 5 (b) shows the result of the Cut Cell method. It is evident that the molten metal charging behavior is different and that the collision part of the molten metal that can cause gas porosity and the final void regions are different. The shape of the smooth circle is created into a stair-form due to the Cartesian coordinates, and the molten metal that should flow along the curve meets an obstacle at the stairs of the computation grid, and thus, the molten metal does not flow along the circle and 


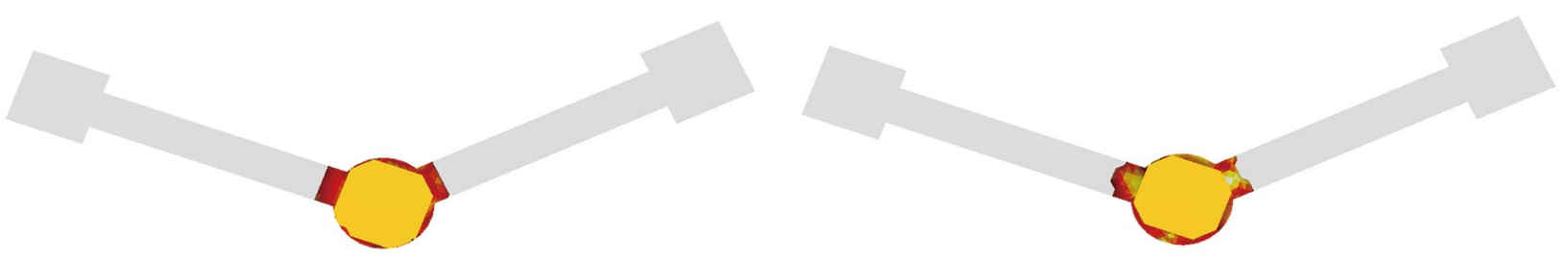

(1) $22 \%$ filled
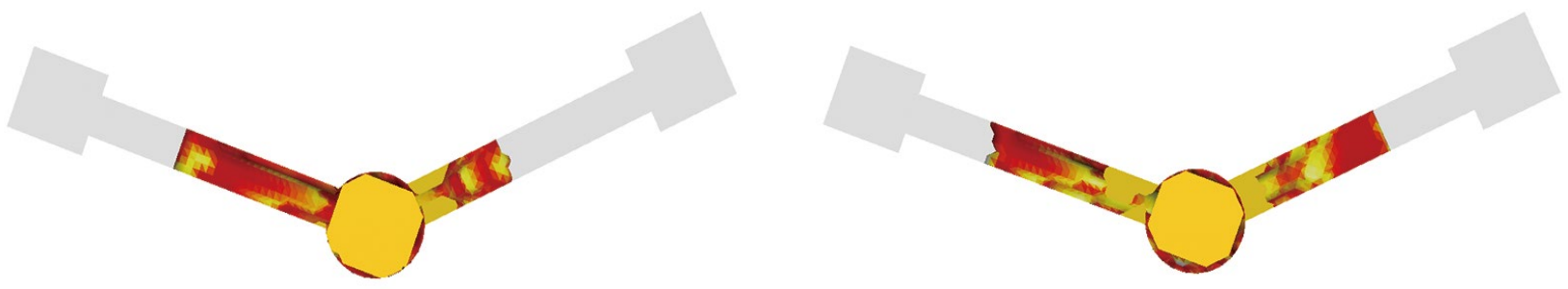

(2) $30 \%$ filled
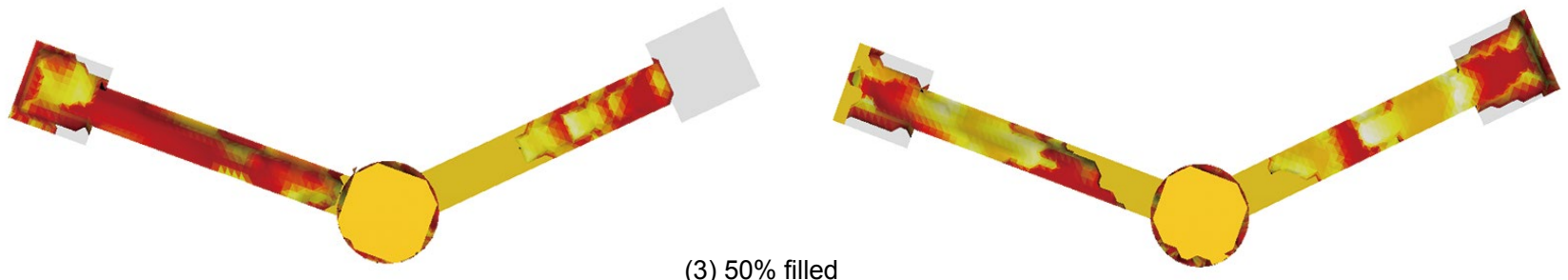

(a) Traditional method

(b) Cut Cell method

Fig. 4: Filling patterns in z-cross section
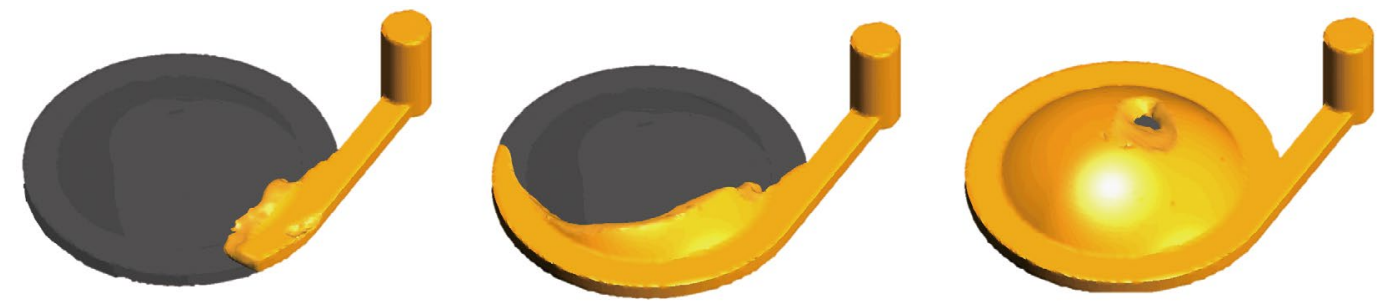

(a) Results of traditional method at $20 \%, 40 \%$ and $96 \%$ filled
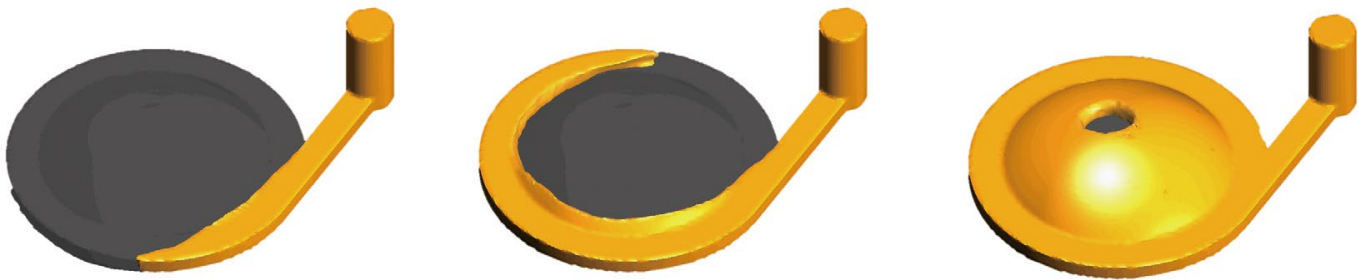

(b) Results of Cut Cell method at $20 \%, 40 \%$ and $96 \%$ filled

Fig. 5: Filling patterns in three dimensions

cover the half-circle. In the case of the Cut Cell method that uses the same coordinates, but calculates the numerical properties through the compensation of momentum equivalent to the amount that the actual casting takes up in the grid, it is found that the molten metal filled the half-circle well regardless of the stair-type grid.

\subsection{Application 1}

The actual casting product cast in the permanent mold was analyzed, as shown in Fig. 6. The material of the molten metal was $\mathrm{A} 356(\mathrm{AC} 4 \mathrm{CH})$ and the material of the mold was $\mathrm{H} 13$ (SKD61). The pouring velocity of the molten metal at the sprue was set at $30 \mathrm{~cm} \cdot \mathrm{s}^{-1}$. 


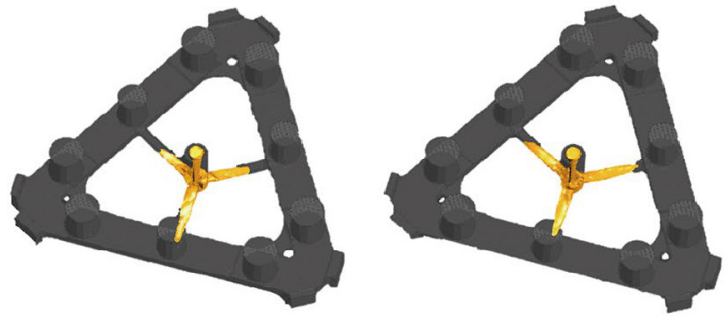

(a) $2 \%$ filled
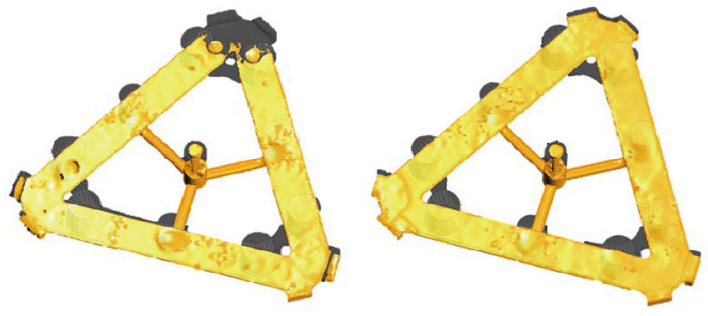

(b) $16 \%$ filled

Fig. 6: Results of traditional method (left) and Cut Cell method (right)

The molten metal that passed the sprue is injected into the mold through three runners. Each runner is set at the same angle. The grid was created by placing just one of the three runners vertically on the coordinates. The other two runners have slopes on the coordinates so a reduction in velocity due to momentum loss here can be expected.

Figure 6 shows the analysis results of the traditional and Cut Cell method. The results of the molten metal filling rate $2 \%$ and $16 \%$ were applied in the actual shape mapping. It is possible to see that the velocity of the molten metal at the sloped runner on the coordinates is slow [Fig. 6(a)]. When filled to $16 \%$, unlike the other two points, the molten metal at the corners of the casting between the sloped runners on the coordinates, are not filled. Meanwhile, when looking at Fig. 6(b) (right), which is the result of using the Cut Cell method, it is seen that the molten metal is filled evenly over the three runners.

\subsection{Application 2}

The last analysis was on the actual casting product - housing, and it was analyzed using the sand gravity casting process. The material of the molten metal was $\mathrm{SCH} 2$ and the material of the mold was furan resin bonded sand. The pouring velocity was set at $50 \mathrm{~cm} \cdot \mathrm{s}^{-1}$. In order to obtain sound casting products, it is advisable that the molten metal fill in the cavity simultaneously through the ingate. This product has a curved runner and three gates inclined to the axis and therefore, the distorted shape on Cartesian coordinates is represented after creating the grids.

Figure 7 shows the results at the molten metal filling rate of $4 \%$ and $8 \%$. When a flow analysis is made in the Cartesian coordinates without the Cut Cell method, there will be stagnation where the molten metal cannot flow smoothly due to the stair-form, unlike the actual runner shape. Due to the excessive pressure caused by stair-form in the runner, the velocity will drop and the molten metal will flow into the mold in the order of the ingates. In Fig. 7 (a), which is the analysis result of the traditional method, the results of the molten metal filling rate of $4 \%$ shows that the molten metal first flows through the ingate, near the gate. While, for the one with the Cut Cell method, it can be seen that at the three ingates, the molten metal is simultaneously injected into the cavity. When using the traditional method at an $8 \%$ molten metal filling rate, the molten metal filling time is $4.016 \mathrm{~s}$, while it is $3.319 \mathrm{~s}$ for the Cut Cell method. The pressure overload due to the stair-form at the runner leads to reduced velocity, thus causing it to take longer to fill with molten metal.

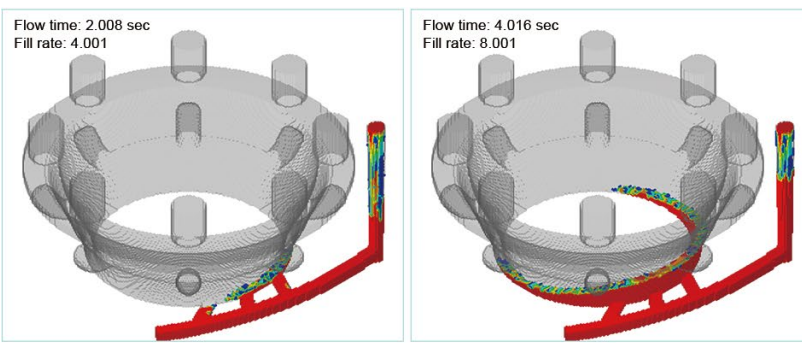

(a) Results of traditional method

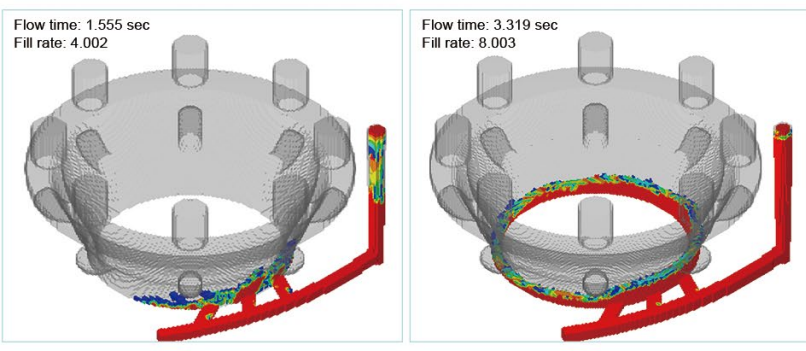

(c) Results of Cut Cell method

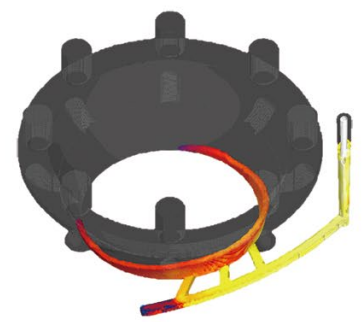

(b) Actual shape of (a), $8 \%$ filled

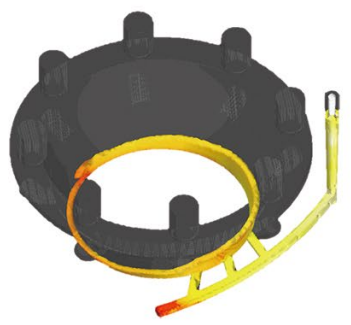

(d) Actual shape of (c), $8 \%$ filled

Fig. 7: Filling results at filling rate of $4 \%$ and $8 \%$ 


\section{Conclusions}

Application of the body-fitted coordinates in casting flow analysis can bring about high-quality analysis results. Despite this, there are difficulties in use due to the difficulty and efficiency issues when creating grids. Meanwhile, because it is relatively quicker and easier to generate grids, Cartesian coordinates were mainly used in the casting processes. However, problems occur as this cannot properly represent the shapes and so it is impossible to acquire the proper analysis results. Normally, many grids are generated to express well the shape of the model to be analyzed in Cartesian grid systems. However, this grid system still has stair grids. It cannot fundamentally resolve problems caused due to stair grids. In casting flow analysis, it is important to predict the collision part and final filling region of the molten metal depending on the filling behavior to better prevent defects, and therefore, it is important to calculate accurately the fluid flow simulation.

In the Cartesian coordinate systems, we compared the traditional method and the Cut Cell method. When using the Cut Cell method, it was confirmed that there was less loss in momentum due to a distortion of shapes in the Cartesian grids and a reduction in loss of velocity in the slopes. In the case of the symmetry geometries, it is confirmed that the analysis result is symmetrical. The result of the conventional method, on the other hand, is not symmetrical due to the inclined shape on the coordinate axis. In addition, it is confirmed through analyzing the actual casting products the Cut Cell method works well.

\section{References}

[1] Delanaye M, Patel A, Leonard B, et al. A Preview Approach to Force Control of Robot Manipulators. ECCOMAS CFD 2001, Institution of Mathematics and Its Application, Southern-onSea, UK, 2001.

[2] Ingram D M, Causon D M, and Mingham C G. Developments in Cartesian cut cell methods. Mathematics and Computers in Simulation, 2003, 61: 561-572.

[3] Hong C P, Lee S Y, and Song K. Development of a new simulation method of mold filling based on a body-fitted coordinate system. ISIJ International, 2001, 41(9): 999-1005.

[4] Lee S Y, Nam N H, Roh D H, et al. Mold filling simulation based on the body fitted coordinate system in an unstructured grid system. In: Proc. 11th Int. Conf. on Modeling of Casting, Welding and Advanced Solidification Processes, Opio, France, 2006: 95-102.

[5] Tucker P G and Pan Z. A Cartesian cut cell method for incompressible viscous flow. Applied Mathematical Modeling, 2000, 24: 591-606.

[6] Lin P. A fixed-grid model for simulation of a moving body in free surface flows. Computers \& Fluids, 2007, 36: 549-561.

[7] Lorensen W E, Clien H E. Marching cubes: A high resolution 3D surface construction algorithm. In: Proc. Int. Conf. Computer Graphics \& Interactive Technique, 1987: 163-169.

[8] Nichols B D, Hirt C W, and Hotchkiss R S. SOLA-VOF: A solution algorithm for transient fluid flow with multiple free boundaries. Tech. Report LA-8355: Los Alamos Scientific Laboratory, 1980. 